\title{
Fluorescent nanosensor designing via hybrid of carbon dots and post- imprinted polymers for the detection of ovalbumin
}

\author{
Xiaoyan Wang ${ }^{\mathrm{a}, *}$, Shuangmei $\mathrm{Yu}^{\mathrm{c}}$, Jiangru Wang ${ }^{\mathrm{b}}$, Jialuo $\mathrm{Yu}^{\mathrm{b}}$, Maryam Arabi $^{\mathrm{b}}$, Longwen $\mathrm{Fu}^{\mathrm{b}}$, \\ Bowei $\mathrm{Li}^{\mathrm{b}}$, Jinhua $\mathrm{Li}^{\mathrm{b}}$, Lingxin $\mathrm{Chen}^{\mathrm{a}, \mathrm{b}, * *}$ \\ ${ }^{a}$ School of Pharmacy, Binzhou Medical University, Yantai, 264003, China \\ ${ }^{\mathrm{b}}$ CAS Key Laboratory of Coastal Environmental Processes and Ecological Remediation, Yantai Institute of Coastal Zone Research, Chinese Cademy of Sciences, Yantai, \\ 264003, China \\ ${ }^{\mathrm{c}}$ Radiotherapy Ward, Yantai Yuhuangding Hospital, Yantai, 264000, China
}

\section{A R T I C L E I N F O}

\section{Keywords:}

Ovalbumin

Post imprinting

Fluorescein isothiocyanate

Protein recognition

Ratiometric fluorescence

\begin{abstract}
A B S T R A C T
We reported a facile strategy to assemble a ratiometric nanosensor for the ovalbumin (OVA) fluorescence determination and meanwhile it can be utilized for selective visual identification by naked eyes with fluorescent test papers under $365 \mathrm{~nm}$ UV lamp. The nanosensor was prepared through simply mixing blue color carbon dots (CDs) and green color core-shell imprinted polymers. Blue CDs were used directly as the internal reference without participating in the imprinting process and modified molecularly imprinted polymers (MIPs) were synthesized by post-imprinting, using fluorescein isothiocyanate (FITC) as fluorescence enhanced signal. Upon the addition of different concentrations of OVA, the fluorescence intensity of FITC was enhanced, while the fluorescence intensity of CDs was almost unchanged, leading to a detection limit as low as $15.4 \mathrm{nM}$. Accordingly, the fluorescence color was gradually changed from blue to dark olive green to green with naked eyes observation. Moreover, the ratiometric nanosensor was successfully applied to detect OVA in the human urine samples with satisfactory recoveries attaining of $92.0-104.0 \%$ with relative standard deviation (RSD) of $3.3-3.9 \%$ and $93.3-101.0 \%$ with RSDs of $2.7-3.8 \%$ for the spiked chicken egg white samples. This strategy reported here opens a novel pathway for biomacromolecule detection in real applications and can realize the visual observation on fluorescent test papers.
\end{abstract}

\section{Introduction}

Glycoproteins, which belong to glycan-conjugated proteins, play key functions in molecular recognition, cell adhesion, immune response and signal transduction [1-3]. Moreover, many glycoproteins are proposed as disease biomarkers and therapeutic targets, since diverse diseases closely correlate with abnormal structural changes and expression of glycoproteins [4]. Ovalbomin (OVA), the most common glycoprotein in egg white, has been used as a model of protein in immunoassay and allergy studies [5,6]. Thus, a series of articles have been reported about OVA assay in fetal bovine serum, human serum and eggs [7-10]. However, because of the low abundance of glycoproteins in complex biological samples and serious matrix disturbance, precise and specific analysis of these kinds of biomolecules is still a great challenge. Hence, the withdrawal of the macromolecule target from the complicated samples is a vital task. Various techniques have been extended for glycoprotein recognition and amongst them, molecularly imprinting technology has succeeded in raising attention owing to remarkable throughput, excellent specificity, cost-effectiveness, and controllable structure.

Molecularly imprinted polymers (MIPs), with specific recognition sites matching the shape, size and functional groups of the template molecules, can selectively recognize and enrich target analytes, which have been applied in a wide range of fields [11]. In contrast with small molecule imprinting, protein imprinting is still sophisticated because of its large size, water solubility, environmental variability and structural complexity. Sol-gel based macromolecule imprinting is an influential method for the preparation of superior MIPs in aqueous media at mild conditions [12]. Meanwhile, post imprinting modification is an innovative approach for producing MIPs by further functionalities to make a mature sorbent to attain higher efficiency. Thus, the combination of sol-gel and post imprinting methods can interestingly promote

\footnotetext{
* Corresponding author.

** Corresponding author.

E-mail addresses: wangxy@yic.ac.cn (X. Wang), lxchen@yic.ac.cn (L. Chen).
} 
MIPs for selective capturing proteins. On the other hand, with the advances of nanotechnology, various versatile excellent nanomaterials for diverse kinds of applications are constantly emerging, such as carbon dots (CDs), quantum dots (QDs), graphene QDs, gold nanoclusters (AuNCs) and so on. Recently, the utilization of fluorescent nanomaterials in the imprinting technology has developed rapidly and fluorescence detection has evolved from early single fluorescence response to ratiometric response. As we know, the change of single wavelength emission intensity can be interfered by the stability of excitation source, environmental change and the local concentration of the fluorescent probe $[13,14]$. Ratiometric fluorescence method possesses self-referencing ability by measuring two emission peaks ratio, eliminating the above unwanted effects and improving sensitivity and accuracy [15]. In addition, ratiometric fluorescence can provide distinct color changes with the increase of target analyte under the UV lamp.

Generally, constructing of ratiometric fluorescence sensing systems include two strategies. One is to build ratiometric fluorescence based on fluorescence resonance energy transfer (FRET). This needs to select a suitable donor and receptor. Besides, it is important to control the distance between the donor and receptor in the imprinting process which if properly selected pledges the specificity $[16,17]$. Another is to construct reference type ratiometric fluorescence that in this strategy, a sensitive to target analyte is regarded as a response signal and the other one is not affected by the analyte, remaining unchanged and used as a reference signal. This construction method is similar to the analysis method with an internal standard that has an auto-calibration impression to grow the detection process with high accuracy, minimized the matrix impact. In the imprinting process, how to keep the reference fluorescence intensity unchanged is a critical problem. Usually, the fluorescent materials (QDs, carbon dots, AuNCs, etc.) as reference materials can be isolated from the target molecules by embedding method. In one study our group used red QDs embedded in silica nanoparticles as a core and NBD as a signal unit, to prepare core-shell MIPs for the ratio detection of 2,4-dichlorophenoxyacetic acid (2,4-D) [18]. Numerous works have been employed embedding method to make the fluorescence intensity of reference materials constant [19-21]. Despite the satisfactory efficiency of this procedure, several synthesis steps and tedious optimization are needed. This inspired us to further extend the construction strategy.

Herein, we developed the assembly of a ratiometric nanosensor by the combination of nanomaterials: blue CDs directly as the internal reference without participating in the imprinting process, and core- shell imprinted polymers synthesized by post-imprinting to enhance the fluorescence signal. The core-shell imprinted polymers were composed of $\mathrm{SiO}_{2}$ nanoparticles as support, 3-aminopropyltriethoxysilane (APTES) as a functional monomer to react with the isothiocyanate group of FITC to form a stable thiourea bond, tetraethyloxysilane (TEOS) as cross-linker and FITC as fluorescence signal amplifier. Ovalbumin (OVA) was acted as a model protein to validate the proof-ofprinciple of our strategy. By exposing OVA to MIPs@FITC at the presence of CDs, the fluorescence intensity of FITC increased, while the fluorescence intensity of CDs had a slight variation. With the increase of OVA concentration, a continuous fluorescence color changed from blue to dark olive green to green on test strips by naked eyes, providing a facile way for OVA identification.

\section{Experimental}

\subsection{Reagents and materials}

Citric acid (CA), dimethylsulfoxide (DMSO), ethylenediamine, ethanol, tetraethoxysilane (TEOS), ammonium hydroxide (25\%), methanol, acetic acid, and phosphate buffered saline (PBS) were bought from Sinopharm Chemical Reagent Co. Ltd. (Shanghai, China). Ovalbumin (OVA), bovine hemoglobin (BHb), lysozyme (Lyz), bovine serum (BSA), 3-aminopropyltriethoxysilane (APTES) and fluorescein isothiocyanate (FITC) were supplied by Sigma-Aldrich (Shanghai, China). Phycocyanin (PC) was kindly provided by Shandong Oriental Ocean Co. (Yantai, China). Ultrapure water of $18.2 \mathrm{M} \Omega$ specific resistance (Millipore, USA) was used in all experiments.

\subsection{Instrumentation}

Fluorescence spectra and UV-Vis absorption were measured on a Fluoromax-4 spectrofluorometer (Horiba Scientific) and NanoDrop 2000/2000C (Thermo Fisher Scientific, Waltham, MA), respectively. A transmission electron microscope (TEM, JEM-1230, operating at $100 \mathrm{kV}$ ), high resolution TEM (HRTEM, JEM-2100, operating at $200 \mathrm{kV}$ ) and scanning electron microscope (SEM, Hitachi S-4800 FESEM, operating at $5 \mathrm{kV}$ ) were used to observe the morphology of nanomaterials. Dynamic light scattering (DLS) measurements were determined by a Malvern Zetasizer Nano-ZS90 (ZEN3590, UK). FT-IR spectra were performed using a Thermo Nicolet FT-IR spectrometer (USA). Elemental analysis was carried by a Vario Micro-cube elementary analyzer (Elementar Company, Germany).

\subsection{Preparation of $C D s$}

Blue CDs were synthesized by a hydrothermal method according to the previous report [22]. (with synthesis details in Supporting Information).

\subsection{Preparation of OVA fluorescent imprinted nanoparticles (MIPs@FITC)}

$\mathrm{SiO}_{2}$ nanoparticles were prepared as our previous report [16]. (with synthesis details in Supporting Information). $20.0 \mathrm{mg}$ OVA and $30 \mu \mathrm{L}$ APTES were added into $20 \mathrm{~mL}$ of ultrapure water containing $0.5 \mathrm{~mL}$ of $20.0 \mathrm{mg} \mathrm{mL}{ }^{-1} \mathrm{SiO}_{2}$. After stirring for $1 \mathrm{~h}, 50 \mu \mathrm{L}$ TEOS and $50 \mu \mathrm{L}$ $\mathrm{NH}_{3} \cdot \mathrm{H}_{2} \mathrm{O}$ were added and the whole reaction system kept stirring for $24 \mathrm{~h}$. The precipitate was centrifuged and washed by methanol/acetic acid $(9: 1, v / v)$ to elute OVA until no OVA was detected in the elution solution, and then washed with ethanol three times. Subsequently, the MIPs were post-conjugated with $1 \mathrm{~mL}$ of $10.0 \mathrm{mg} \mathrm{mL}^{-1}$ FITC (solvent, DMSO) for $2 \mathrm{~h}$. Then ethanol was used to remove excess unreacted FITC. Finally, the resultant MIPs@FITC was stored in water for further experiments. As a control, the non-imprinted polymers (NIPs@FITC) were prepared by the same procedure in the absence of the template OVA.

\subsection{Assembly of the molecularly imprinted ratiometric fluorescence nanosensor (designated C-MIPs@FITC)}

To construct the C-MIPs@FITC nanosensor, $50 \mu \mathrm{L}$ of CDs solution (4.7 $\mu \mathrm{g} \mathrm{mL}^{-1}$ ) and $50 \mu \mathrm{L}$ of MIPs@FITC solution (3.165 $\mathrm{mg} \mathrm{mL}^{-1}$ ) were mixed in $0.9 \mathrm{~mL}$ PBS solution $\left(0.01 \mathrm{~mol} \mathrm{~mL}^{-1}\right)$. The fluorescence spectrum of C-MIPs@FITC was recorded under a single wavelength excitation at $390 \mathrm{~nm}$. The construction schematic of the C-MIPs@FITC sensor is shown in Fig. 1.

\subsection{Preparation of chicken egg white sample}

The chicken egg white sample was prepared as follows. The chicken egg white was separated from the egg yolk and then was diluted 50-fold with $10 \mathrm{mM}$ PBS ( $\mathrm{pH}$ 8.0). After the solution was under ultrasound sonication for $30 \mathrm{~min}$, it was filtered three times by using a $0.2 \mu \mathrm{m}$ membrane filter and then was further diluted in the linear range for future detection.

\subsection{Preparation of fluorescent test papers}

The filter paper-based strip (Whatman chromatography No. 1 paper, GE) was printed by a wax printer (XEROX Phaser $8560 \mathrm{DN}$ ) to obtain a 




Fig. 1. Schematic illustration of the synthesis and the assembly of C-MIPs@FITC nanosensor.

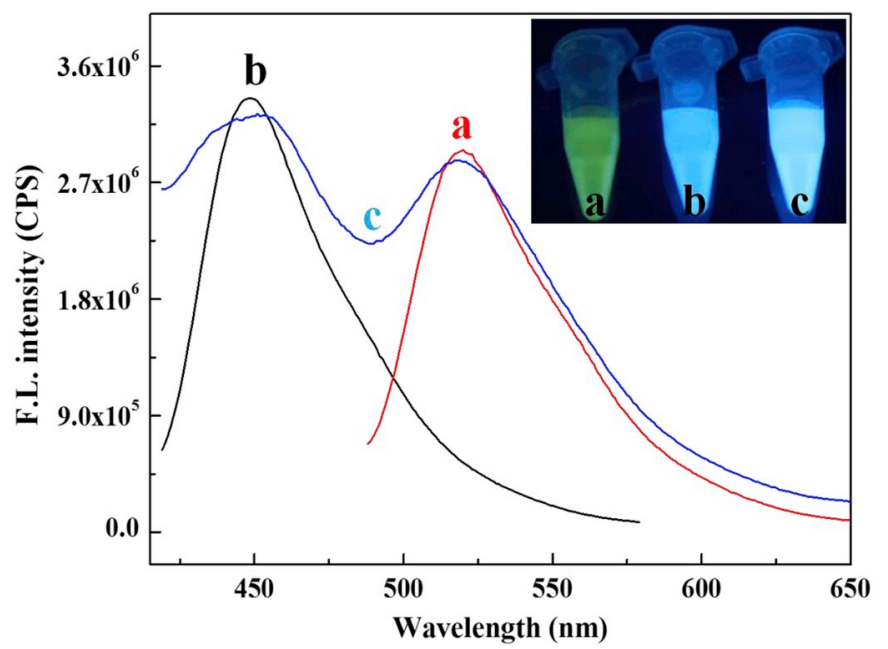

Fig. 2. Fluorescence emission spectra of (a) MIPs@FITC, (b) CD dots, and (c) CMIPs@FITC.

line of spots (diameter: $0.5 \mathrm{~cm}$ ). The wax-patterned paper was put into the oven at $120{ }^{\circ} \mathrm{C}$ for $120 \mathrm{~s}$, so that the wax can penetrate through the whole paper completely and thus form hydrophobic barriers. For OVA detecting, different concentrations of OVA solution $(10 \mu \mathrm{L})$ were added into the C-MIPs@FITC nanosensor, and then $3 \mu \mathrm{L}$ of the mixed solution was dropped onto per spot for further observation. For OVA testing in human urine, varying concentrations of OVA spiked samples (PBS buffer pH 8.0) into 100-fold diluted urine were dropped in the C-MIPs@ FITC nanosensor, and then $3 \mu \mathrm{L}$ of the mixed solution was dropped onto per spot. For OVA testing in chicken egg white sample, diluted chicken egg white samples were spiked with OVA at the concentrations of 0.3 , 0.5 and $1.0 \mu \mathrm{M}$ by dropping in the C-MIPs@FITC nanosensor, and then the mixed solution was dropped onto per spot. After drying, the color of the test papers was observed and photos were taken with a digital camera (CANON EOS 6D) under a $365 \mathrm{~nm}$ UV lamp.

\section{Results and discussion}

3.1. Assembly and characterization of molecularly imprinted ratiometric fluorescence nanosensor

As seen in Fig. 1, the core-shell MIPs@FITC were prepared through a simple sol-gel process. In detail, $\mathrm{SiO}_{2}$ nanoparticles were used as support, ATPES as functional monomer, OVA as a template, TEOS as cross-linker and $\mathrm{NH}_{3} \cdot \mathrm{H}_{2} \mathrm{O}$ as the catalyst, after polymerization and eluting OVA, plentiful specific binding sites were created on the surface of $\mathrm{SiO}_{2}$ nanoparticles, complementary to OVA terms of functionality and stereochemistry. This phenomenon can be proved according to the physical characterization (SEM images in Fig. 3e and f) and the selectivity experiments (section 3.4. Sensitivity and selectivity of the CMIPs@FITC nanosensor). Then FITC was conjugated to OVA MIPs through post-imprinting treatment since imprinted cavities had amino groups, which can react with the isothiocyanate group of FITC to form thiourea bond. The resultant MIPs@FITC exhibited an emission at $520 \mathrm{~nm}$ with green fluorescence (Fig. 2a). CDs displayed an emission at $454 \mathrm{~nm}$ with blue fluorescence (Fig. 2b). When MIPs@FITC were mixed with CDs in appropriate proportion, the C-MIPs@FITC nanosensor was obtained with two fluorescence emission peaks at 454 and $520 \mathrm{~nm}$ under a single excitation at $390 \mathrm{~nm}$ (Fig. 2c). Since FITC acted as recognition signal and MIPs provided selective recognition sites, OVA can 

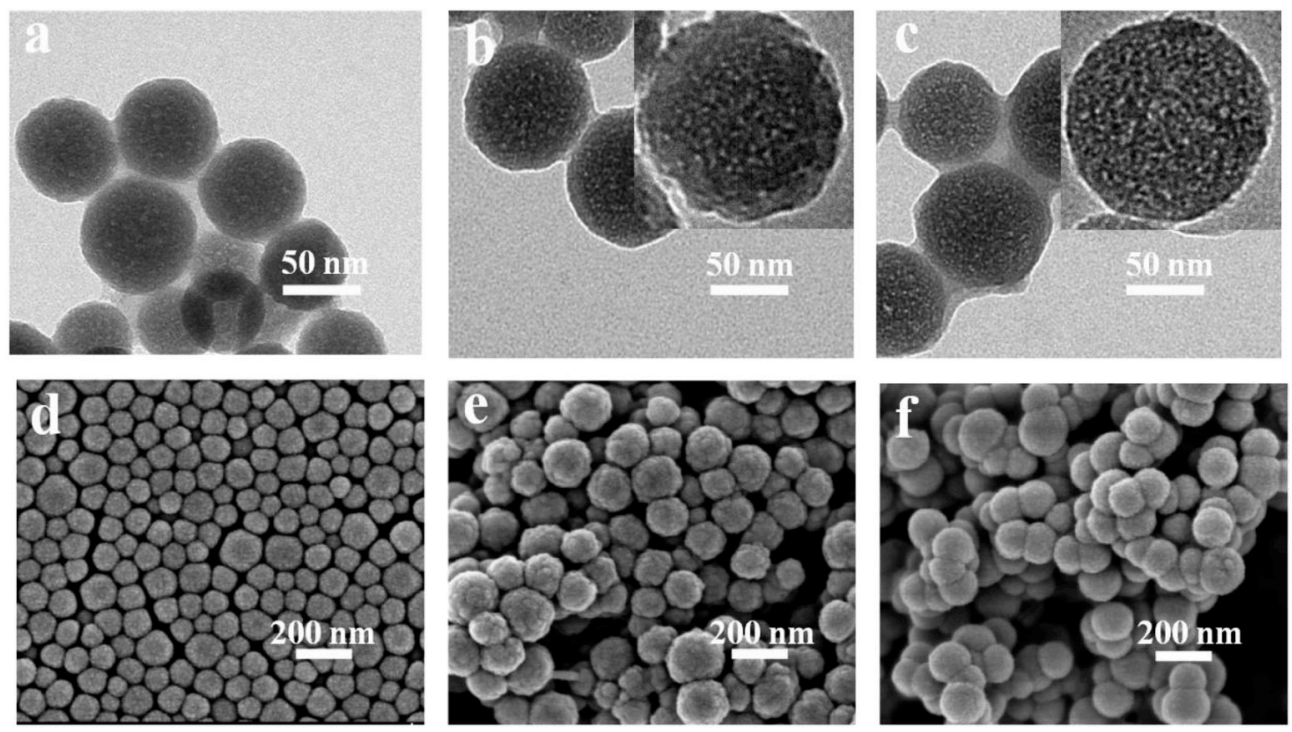

Fig. 3. TEM and SEM images of nanoparticles (a, d) $\mathrm{SiO}_{2}$, (b, e) MIPs@FITC, and (c, f) NIPs@FITC, inset: HRTEM image.

enter into the imprinted cavities, and enhanced the fluorescence intensity of FITC, as seen in Fig. S1. This phenomenon might be attributed to the bounded OVA that changed the hydrophobic/hydrophilic environment condition of the imprinted cavities around FITC, which was in favor of FITC emission and resulted in the fluorescence intensity increasing [23-26]. As seen in Fig. S2, the absorption spectrum of OVA had no spectral overlap with the emission spectrum of FITC. Therefore, fluorescence resonance energy transfer between FITC and OVA was excluded. With the concentration of OVA increased, the green fluorescence of FITC was enhanced, while the blue fluorescence of CDs was almost unchanged and thus acted as a reference signal. Based on this, OVA can be detected by the variation of fluorescence intensity ratios with the fluorescence color change from blue to dark olive green to green.

The size and shape of $\mathrm{SiO}_{2}$, MIPs@FITC, and NIPs@FITC were examined by SEM and TEM. As observed in Fig. 3, the surface of $\mathrm{SiO}_{2}$ nanoparticles was velvety and uniformly dispersed with an average particle size of $70 \mathrm{~nm}$ (Fig. 3a and d). Compared to $\mathrm{SiO}_{2}$ nanoparticles, the particle size of MIPs@FITC and NIPs@FITC was approximately $90 \mathrm{~nm}$ due to the process of polymerization (Fig. 3b, c, e, and f). Noticeably, MIPs@FITC showed a highly rough imprinting layer (Fig. 3b), by comparison, the surface of NIPs@FITC particles was still smooth (Fig. 3c), suggesting that a large number of imprinted cavities were formed on the surface of MIPs, which was a benefit for rebinding of OVA. It should be pointed out that, slim imprinted layer benefit from unique advantages viz. more specific sites accessibility, facility masstransfer of the analyte, and also hinder stereo avoidance for the large target protein to entire MIPs' cavities. The size distributions of $\mathrm{SiO}_{2}$, MIPs@FITC, and NIPs@FITC were also displayed by DLS measurement in Fig. S3. The main distribution peak of $\mathrm{SiO}_{2}$ was around $72 \mathrm{~nm}$ and the diameters of MIPs@FITC and NIPs@FITC were mainly around $95 \mathrm{~nm}$. This is consistent with the results of TEM images.

Elemental analysis was carried out to examine N, C, H and S content (Table S1). As shown, $\mathrm{SiO}_{2}$ nanoparticles contain a low amount of $\mathrm{N}$ that is attributed to the residual of ammonium hydroxide. On the other hand, compared to the low $\mathrm{N}$ content of $\mathrm{SiO}_{2}$, more grain $\mathrm{N}$ can be found in MIPs@FITC and NIPs@FITC, suggesting that - $\mathrm{NH}_{2}$ was successfully introduced. As seen in Fig. S3 and Fig. 3, the size of NIPs is more than MIPs and therefore the amount of polymeric layer in NIPs is more than MIPs. However, the vacant location in MIPs' skeleton is more than NIPs due to the template removal. Hence, owing to the higher imprinting cavities of MIPs compared with NIPs, more amount of FITC can be grafted to the MIPs. This phenomenon is proved by higher percentages of S $(0.930 \%), \mathrm{H}(3.482 \%)$, and C (18.043\%) elements in MIPs and suggested successful post-imprinting treatment, and the coarser surface of MIPs in contrast with NIPs (Fig. 3e and f) demonstrated this fact as well.

Furthermore, the successful synthesis of MIPs@FITC and NIPs@ FITC was proved by the existence of expected functional groups in FTIR spectra (Fig. S4). As seen, MIPs@FITC and NIPs@FITC displayed the characteristic peak of amino groups at about $1393 \mathrm{~cm}^{-1}$ and the aliphatic stretching of the $\mathrm{C}-\mathrm{H}$ bond at $2954 \mathrm{~cm}^{-1}$, suggesting that amine groups are in both MIPs@FITC and NIPs@FITC and feasibility interacts with OVA $[27,28]$. The characteristic peak of the benzene ring frame vibration appeared at about $1577 \mathrm{~cm}^{-1}$ and $\mathrm{C}=\mathrm{O}$ stretching vibration of carboxyl at about $1654 \mathrm{~cm}^{-1}$ meant that FITC had been successfully conjugated to MIPs@FITC and NIPs@FITC through post-imprinting treatment. Moreover, the similarity in FT-IR spectra of MIPs@FITC and NIPs@FITC revealed that template spices were removed from MIPs@ FITC completely.

\subsection{Ratio optimal for MIPs@FITC and CDs in the C-MIPs@FITC nanosensor}

The fluorescence peaks intensity at 454 and $520 \mathrm{~nm}$ were attributed to the CDs and MIPs@FITC, respectively. When dispersing both MIPs@ FITC and CDs simultaneously, and the ratio of them was 5:1, the fluorescent color changed only from light green to green upon the addition of OVA (Fig. S5A). As the proportion decreases to 1:1, wide colors from blue to dark olive green to green were obtained. Continued to increase the amount of $\mathrm{CDs}$ and the ratio reached 1:2, the color changed from blue to light blue (Fig. S5B). For the optimal OVA visualization, the C-MIPs@FITC system consisted of 1:1 MIPs@FITC and CDs.

\subsection{Condition optimization for the C-MIPs@FITC nanosensor}

The fluorescence performance of the C-MIPs@FITC nanosensor was mainly influenced by the parameters, like $\mathrm{pH}$, stability and response time, which were optimized as follows. The influence of $\mathrm{pH}$ was evaluated by the value of $\left(F-F_{0}\right) / F_{0}$, using as the index of enhancing efficiency. $F_{0}$ and $F$ represented the fluorescence emission intensity of FITC in the absence and presence of the OVA, respectively. As shown in Fig. $\mathrm{S} 6 \mathrm{~A}$, in the $\mathrm{pH}$ range of 5.0-9.0, the enhancing efficiency increased and then decreased. At $\mathrm{pH}$ 8.0, the enhancing efficiency reached a maximum. As we know, under high or low $\mathrm{pH}$, OVA may be denatured. 

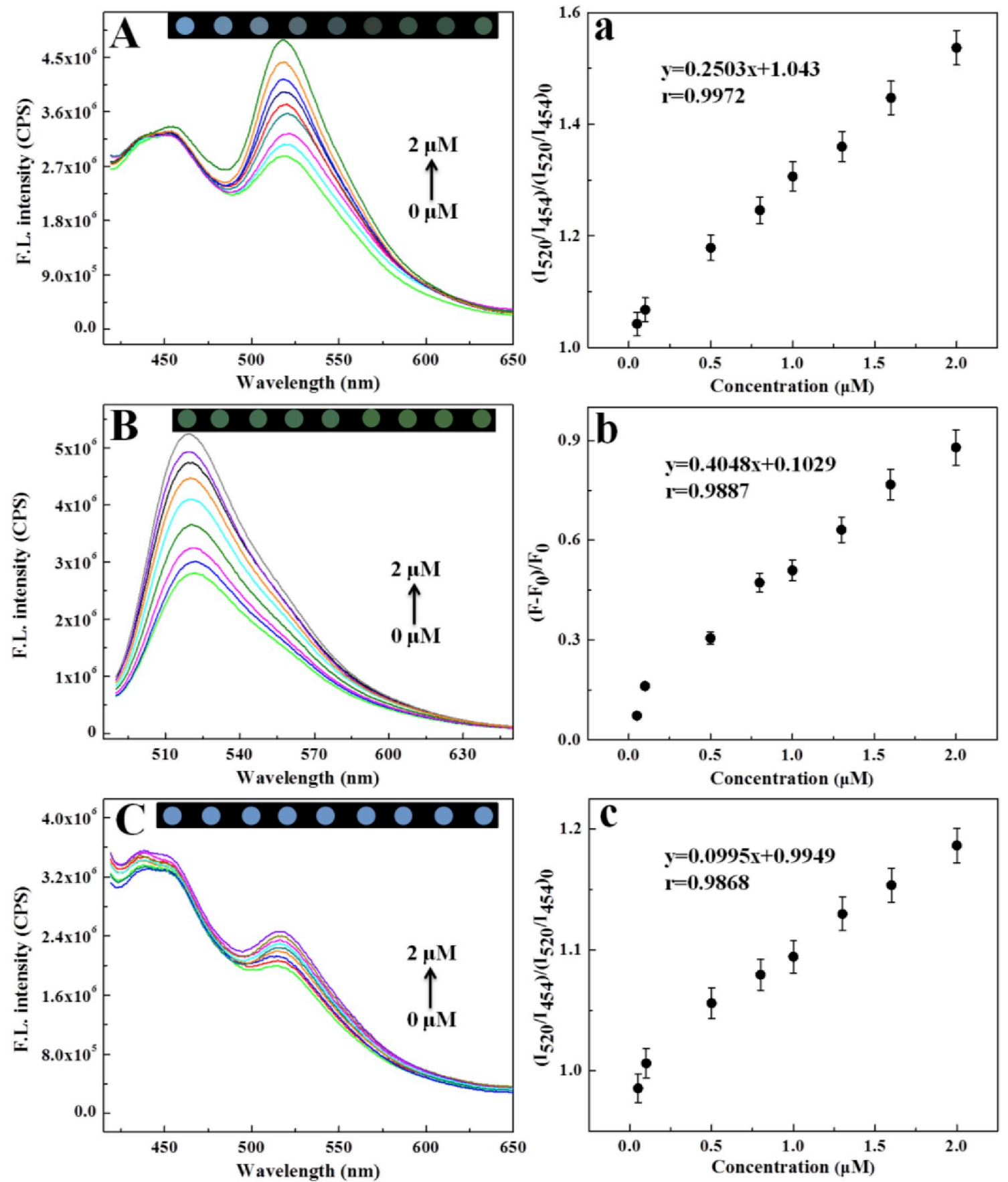

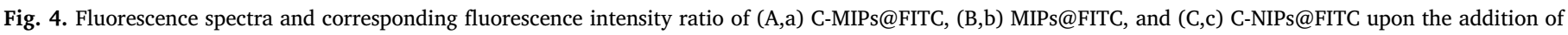
different amounts of OVA; The ratio of MIPs@FITC or NIPs@FITC and CDs is 1:1.

Besides, under acidic conditions, the emission intensity of FITC was greatly reduced, mainly due to the dominant formation of neutral and cationic forms, which gave lower intensity fluorescence [29]. On the other hand, the $\mathrm{NH}_{2}$ groups of APTES at $\mathrm{pH}$ lower than 6.0, induced by positive charge $\left(\mathrm{NH}_{3}{ }^{+}\right)$that leads to the poor affinity toward OVA. At a $\mathrm{pH}$ higher than 7.0, the $\mathrm{NH}_{2}$ groups are in neutral form, the binding affinity of MIPs@FITC to target species increased. Therefore, the pH value was fixed at 8.0 for the following work.

The stability of the C-MIPs@FITC nanosensor was measured by fluorescence spectra. The fluorescence intensity ratios $\left(I_{520} / I_{454}\right)$ was not significantly changed over $2 \mathrm{~h}$ (Fig. S6B), indicating that the system had supreme fluorescence stability.

In addition, to investigate the kinetics of the C-MIPs@FITC nanosensor, the fluorescence intensity at $520 \mathrm{~nm}$ was investigated at different time intervals when adding OVA (Fig. S6C). As seen, the fluorescence intensity increased immediately with the addition of $1 \mu \mathrm{M}$ OVA and then kept stable in $10 \mathrm{~min}$. Thus, $10 \mathrm{~min}$ was set for the incubation time.

\subsection{Sensitivity and selectivity of the C-MIPs@FITC nanosensor}

The sensitivity performance of the C-MIPs@FITC nanosensor was evaluated by adding the different concentrations of OVA. As seen in Fig. 4A, the fluorescence intensity of FITC gradually increased while that of CDs remained constant with increased OVA concentration. Thanks to the fluorescence intensities increasing and stability, the 


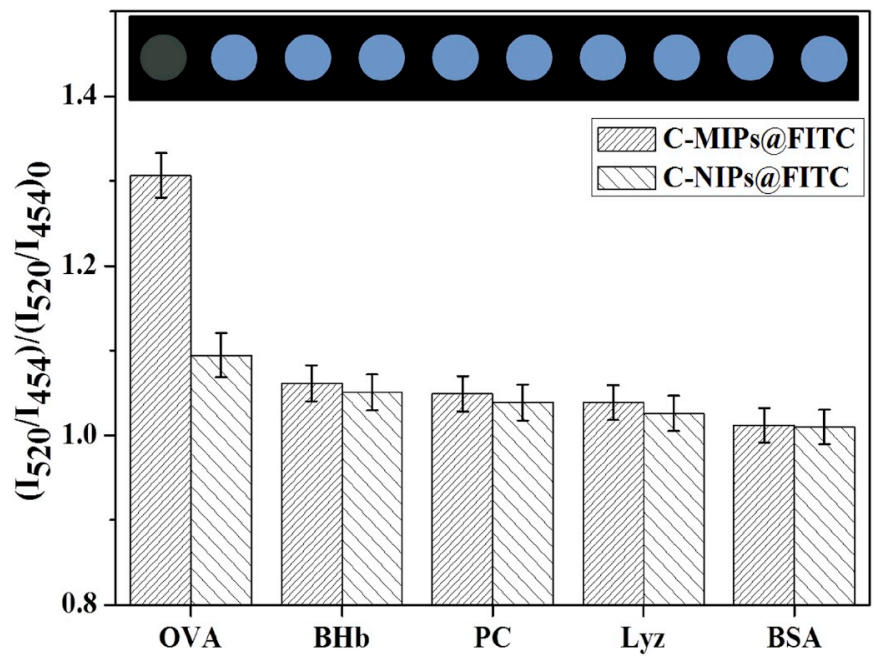

Fig. 5. Selectivity of C-MIPs@FITC nanosensor and C-NIPs@FITC for OVA over other proteins including BHb, PC, Lyz and BSA at concentration of $1 \mu \mathrm{M}$ and the inset showed the corresponding fluorescence color change of the test paper from left to right. The concentration of C-MIPs@FITC or C-NIPs@FITC was $0.16 \mathrm{mg} \mathrm{mL}^{-1}$; excited light, $390 \mathrm{~nm}$; silt widths of excitation and emission, $6 \mathrm{~nm}$. (For interpretation of the references to color in this figure legend, the reader is referred to the Web version of this article.)

fluorescence intensity ratio changed, resulting in the color change from blue to dark olive green to green, which can be easily observed by the naked eyes. A good linear relationship was displayed between the ratio of the intensities $\left(I_{520} / I_{454}\right)$ and the concentration of OVA ranging from 0.05 to $2 \mu \mathrm{M}$ with correlation coefficient $\mathrm{r}=0.9972$ (Fig. 4a). The detection limit was calculated to $15.4 \mathrm{nM}$ based on $3 \sigma / s$, where $\sigma$ represents the standard deviation of the blank measurements and $s$ meant the slope of the linear calibration.

To demonstrate the superiority of the C-MIPs@FITC nanosensor in sensitivity and visualization, the single emission peak of MIPs@FITC was also measured and is shown in Fig. 4B. The linearity trend of the MIPs@FITC sensor was the same as C-MIPs@FITC while the LOD was $25.1 \mathrm{nM}$ that demonstrates lower sensitivity of MIPs@FITC. By increasing the concentration of OVA, the color change was not obvious and can not be distinguished by naked eyes. By comparing, the ratiometric nanosensor showed higher sensitivity and more remarkable color changes for easy naked eyes observation.
The sensitivity of the C-NIPs@FITC was also investigated as shown in Fig. 4C. Compared to C-MIPs@FITC, when the same concentration of OVA was analyzed, the fluorescence intensity of FITC increased slightly, leading to the color change unobvious (Fig. 4C, inset). According to Fig. 4, a high imprinting factor of 2.5 was obtained based on the ratio of the slopes of linear relationships of MIPs and NIPs. The MIPs had superior sensitivity than the NIPs owing to the specific imprinted recognition sites on the surface, so the OVA have more chance to combine for recovering the fluorescence intensity of FITC. Therefore, the CMIPs@FITC based on dual emitting ratiometric fluorescence nanosensor performed high sensitivity for OVA detection and can be easily recognized by the naked eyes.

Meanwhile, the selectivity of C-MIPs@FITC nanosensor was further investigated by adding the same concentrations of OVA and other proteins (BHb, PC, Lyz, and BSA). As shown in Fig. 5, OVA led to a significantly high fluorescence enhancing of FITC in the ratio C-MIPs@ FITC nanosensor and olive green fluorescence color was present. Whereas, other proteins didn't present obvious changes in fluorescence intensity ratios $\left(\mathrm{I}_{520} / \mathrm{I}_{454}\right)$ and the blue colors were unchanged. It can be explained by creating a large number of specific imprinted recognition sites into the C-MIPs@FITC nanosensor that perfectly matched with OVA through the hydrogen bond interaction between the amine groups and OVA. OVA can easily recombine with the tailor-made binding sites, but other proteins had less chance to access due to the big differences from OVA in shape, size, and structure. In addition, C-NIPs@FITC was also observed to have similar color changes and slightly fluorescence intensity ratios for OVA and the other four proteins. Hence, the CMIPs@FITC nanosensor has shown a great selectivity for the detection of OVA.

\subsection{Application of the C-MIPs@FITC nanosensor to real samples}

In order to evaluate the utility of the formed C-MIPs@FITC nanosensor, human urine which was randomly taken from one of three healthy volunteers and chicken egg white were used as the real samples. The human urine was diluted 100 -fold and spiked with different concentrations $(0.3,0.5,1.0 \mu \mathrm{M})$ of OVA. The analytical results showed that no OVA was found in human urine and also the endogenous OVA concentration was calculated to be $0.21 \mu \mathrm{M}$ in the egg white. Based on spectrofluorometer determination, satisfactory recoveries of 92.0-104.0\% with relative standard deviation (RSD) of $3.3-3.9 \%$ for the spiked urine samples and $93.3-101.0 \%$ with RSDs of $2.7-3.8 \%$ for the spiked chicken egg white samples were attained, respectively

Table 1

Results for the determination of OVA in human urine samples and chicken egg white using the C-MIPs@FITC nanosensor.

\begin{tabular}{|c|c|c|c|c|}
\hline Sample & Added $(\mu \mathrm{M})$ & Found $(\mu M)$ & Fluorescence color & $\begin{array}{c}\operatorname{Recovery}^{\mathrm{a}} \pm \text { RSD } \\
(\%)\end{array}$ \\
\hline \multirow{3}{*}{ human urine } & 0.3 & 0.29 & & $96.6 \pm 3.5$ \\
\hline & 0.5 & 0.46 & & $92.0 \pm 3.3$ \\
\hline & 1.0 & 1.04 & & $104.0 \pm 3.9$ \\
\hline \multirow[t]{3}{*}{ chicken egg } & 0.3 & 0.49 & & $93.3 \pm 3.4$ \\
\hline & 0.5 & 0.70 & & $98.0 \pm 2.7$ \\
\hline & 1.0 & 1.22 & & $101.0 \pm 3.8$ \\
\hline
\end{tabular}

a Average value from three individual experiments. 
(Table 1). Meanwhile, the fluorescent test papers presented the obvious color responses to the different concentrations of OVA. Promisingly, the C-MIPs@FITC nanosensor showed remarkable merits of application and reliability for OVA detection.

\subsection{Method performance comparison}

The performance of the developed fluorescence methods for intensity increase determination was compared as listed in Table S2. Sometimes, indirect fluorescence detection methods can provide innovative ways to efficiently detection. The OVA detections were few, like Ref. 30, 31. In Ref. 30, the OVA-antibody modified NOP-CDs were quenched by GO due to the FRET effect. The introduced OVA competed to displace the GO by special binding with the anti-OVA, resulting in the recovery of the fluorescence with a good detection limit [30]. However, there was only one emission peak without obvious color change. About Ref. 31, the authors ingeniously used protein bindinginduced surfactant aggregation variation for both pepsin and ovalbumin egg detection [31]. But the specificity for OVA needed to be improved. Nowadays, there are many ways to fabricate the MIPs with fluorescence enhancement for detection based on the specific interaction between the templates and the recognition cavities [18,32-34]. Although, the responding mechanisms were diverse, like in Ref. 32, 33, the glycoprotein combined with the boronic acid was the key for detection. These ways produced new considerations of indirect fluorescence detection. Additionally, the sensitivity of our present sensor was also compared with other fluorescent sensors, as shown in Table S2. Our LOD is comparable to or lower than that of the former methods. Overall, the present C-MIPs@FITC nanosensor in our study demonstrated excellent sensitivity and selectivity for convenient and visual ratio detection of OVA.

\section{Conclusions}

To summarize, we assembled the ratiometric nanosensor by mixing blue CDs and green core-shell imprinted polymers (MIPs@FITC) for sensitive visualization of OVA. This development strategy was facile and convenient: the CDs did not need any further chemical modification and directly used as the internal reference; MIPs@FITC was prepared by the sol-gel process and then through post-conjugation, providing fluorescence enhanced signal and selective recognition cavities. Moreover, the sensitive and selective detection of OVA can be displayed on fluorescent test papers by naked eyes observation. Consequently, we expect our work will pave the way for the growth of sensing biomacromolecule in biological applications.

\section{Notes}

The authors declare no competing financial interests.

\section{Acknowledgements}

This work was financially supported by the National Natural Science Foundation of China (21804010, 21575159, 41776110, 21876199, 21976209), the Science and Technology Development Plan of Shandong Province of China (2014GGF01074), the Chinese Academy of Sciences President's International Fellowship Initiative (2019PC0050), Research Initiation Fund of Binzhou Medical University (BY2019KYQD39), and the Taishan Scholar Project Special Funding (No.ts20190962).

\section{Appendix A. Supplementary data}

Supplementary data to this article can be found online at https:// doi.org/10.1016/j.talanta.2020.120727.

\section{References}

[1] T. Guo, Q.L. Deng, G.Z. Fang, Y.G. Yun, Y.J. Hu, S. Wang, A double responsive smart upconversion fluorescence sensing material for glycoprotein, Biosens. Bioelectron. 85 (2016) 596-602.

[2] X.Y. Tu, P. Muhammad, J. Liu, Y.Y. Ma, S.S. Wang, D.Y. Yin, Z. Liu, Molecularly imprinted polymer-based plasmonic immunosandwich assay for fast and ultrasensitive determination of trace glycoproteins in complex samples, Anal. Chem. 88 (2016) 12363-12370.

[3] R.R. Xing, S.S. Wang, Z.J. Bie, H. He, Z. Liu, Preparation of molecularly imprinted polymers specific to glycoproteins, glycans and monosaccharides via boronate affinity controllable-oriented surface imprinting, Nat. Protoc. 12 (2017) 964-987.

[4] G. Walsh, R. Jefferis, Post-translational modifications in the context of therapeutic proteins, Nat. Biotechnol. 24 (2006) 1241-1252.

[5] T.W. Ng, P.G. Holt, S.L. Prescott, Cellular immune responses to ovalbumin and house dust mite in egg-allergic children, Allergy 57 (2002) 207-214.

[6] R.G. Heine, N. Laske, D.J. Hill, The diagnosis and management of egg allergy, Curr. Allergy Asthma Rep. 6 (2006) 145-152.

[7] K. Sugawara, H. Kuramitz, H. Shinohara, Fabrication of micromagnetic beads with molecular recognition/electron-transfer peptides for the sensing of ovalbumin, Anal. Chim. Acta 958 (2017) 30-37.

[8] J.F. Xie, G.Q. Zhong, C.Q. Cai, C.Y. Chen, X.Y. Chen, Rapid and efficient separation of glycoprotein using $\mathrm{pH}$ double-responsive imprinted magnetic microsphere, Talanta 169 (2017) 98-103.

[9] J. Luo, J. Huang, Y.N. Wu, J. Sun, W. Wei, X.Y. Liu, Synthesis of hydrophilic and conductive molecularly imprinted polyaniline particles for the sensitive and selective protein detection, Biosens. Bioelectron. 94 (2017) 39-46.

[10] T. Saeki, H. Sunayama, Y. Kitayama, T. Takeuchi, Orientationally fabricated zwitterionic molecularly imprinted nanocavities for highly sensitive glycoprotein recognition, Langmuir 35 (2019) 1320-1326.

[11] L.X. Chen, X.Y. Wang, W.H. Lu, X.Q. Wu, J.H. Li, Molecular imprinting: perspectives and applications, Chem. Soc. Rev. 45 (2016) 2137-2211.

[12] Z. Bie, Y. Chen, J. Ye, S. Wang, Z. Liu, Boronate-affinity glycan-oriented surface imprinting: a new strategy to mimic lectins for the recognition of an intact glycoprotein and its characteristic fragments, Angew. Chem. Int. Ed. 54 (2015) 10211-10215.

[13] W. Song, W.X. Duan, Y.H. Liu, Z.J. Ye, Y.L. Chen, H.L. Chen, S.D. Qi, J. Wu, D. Liu, L.H. Xiao, C.L. Ren, X.G. Chen, Ratiometric detection of intracellular lysine and $\mathrm{pH}$ with one-pot synthesized dual emissive carbon dots, Anal. Chem. 89 (2017) 13626-13633.

[14] Q. Yang, J.H. Li, X.Y. Wang, H.L. Peng, H. Xiong, L.X. Chen, Strategies of molecular imprinting-based fluorescence sensors for chemical and biological analysis, Biosens. Bioelectron. 112 (2018) 54-71.

[15] Y. Kim, G. Jang, T.S. Lee, Carbon nanodots functionalized with rhodamine and poly (ethyleneglycol) for ratiometric sensing of $\mathrm{Al}$ ions in aqueous solution, Sens. Actuators, B 249 (2017) 59-65.

[16] X.Y. Wang, J.L. Yu, Q. Kang, D.Z. Shen, J.H. Li, L.X. Chen, Molecular imprinting ratiometric fluorescence sensor for highly selective and sensitive detection of phycocyanin, Biosens. Bioelectron. 77 (2016) 624-630.

[17] H.Z. Lu, S.F. Xu, Visualizing BPA by molecularly imprinted ratiometric fluorescence sensor based on dual emission nanoparticles, Biosens. Bioelectron. 92 (2017) $147-153$.

[18] X.Y. Wang, J.L. Yu, X.Q. Wu, J.Q. Fu, Q. Kang, D.Z. Shen, J.H. Li, L.X. Chen, A molecular imprinting-based turn-on ratiometric fluorescence sensor for highly selective and sensitive detection of 2,4-dichlorophenoxyaceticacid(2,4-D), Biosens. Bioelectron. 81 (2016) 438-444.

[19] S.F. Xu, H.Z. Lu, Ratiometric fluorescence and mesoporous structure dual signal amplification for sensitive and selective detection of TNT based on MIP@QD fluorescence sensors, Chem. Commun. 51 (2015) 3200-3203.

[20] H.Z. Lu, S.F. Xu, Functional monomer-template-QDs sandwich structure for mesoporous structured bovine hemoglobin imprinted ratiometric fluorescence sensor, Talanta 165 (2017) 482-488.

[21] X.Y. Wang, S.M. Yu, W. Liu, L.W. Fu, Y.Q. Wang, J.H. Li, L.X. Chen, Molecular imprinting based hybrid ratiometric fluorescence sensor for the visual determination of bovine hemoglobin, ACS Sens. 3 (2018) 378-385.

[22] X.Y. Huang, Y.J. Zhou, C. Liu, R.L. Zhang, L.Y. Zhang, S.H. Du, B.H. Liu, M.Y. Han, Z.P. Zhang, A single dual-emissive nanofluorophore test paper for highly sensitive colorimetry-based quantification of blood glucose, Biosens. Bioelectron. 86 (2016) 530-535.

[23] S. Aoyagi, T. Miyasaka, Y. Yoshimi, K. Sakai, A new reagentless immunosensor for measuring IgG concentration in human plasma based on fluorescence-enhancement immunoassay, J. Artif. Organs 5 (2002) 60-63.

[24] S. Aoyagi, R. Imai, K. Sakai, M. Kudo, Reagentless and regenerable immunosensor for monitoring of immunoglobulin G based on non-separation immunoassay, Biosens. Bioelectron. 18 (2003) 791-795.

[25] F. You, Y.F. Zhou, X.E. Zhang, Z. Huang, L.J. Bi, Z.P. Zhang, J.K. Wen, Y.Y. Chen, G.B. Jiang, M.H. Zheng, Cell-free bioassay for measurement of dioxins based on fluorescence enhancement of fluorescein isothiocyanate-labeled DNA probe, Anal. Chem. 78 (2006) 7138-7144.

[26] H. Sunayama, T. Takeuchi, Molecularly imprinted protein recognition cavities bearing exchangeable binding sites for postimprinting site-directed introduction of reporter molecules for readout of binding events, ACS Appl. Mater. Interfaces 6 (2014) 20003-20009.

[27] J.X. Wang, L. Gao, D.L. Han, J.M. Pan, H. Qiu, H.J. Li, X. Wei, J.D. Dai, J.H. Yang, H. Yao, Y.S. Yan, Optical detection of $\lambda$-cyhalothrin by core-shell fluorescent 
molecularly imprinted polymers in Chinese spirits, J. Agric. Food Chem. 63 (2015) 2392-2399.

[28] J.X. Wang, H. Qiu, H.Q. Shen, J.M. Pan, X.H. Dai, Y.S. Yan, G.Q. Pan, B. Sellergren, Molecularly imprinted fluorescent hollow nanoparticles as sensors for rapid and efficient detection $\lambda$-cyhalothrin in environmental water, Biosens. Bioelectron. 85 (2016) 387-394.

[29] G.W. Lim, J.K. Lim, A.L. Ahmad, D.J.C. Chan, Fluorescent molecularly imprinted polymer based on navicula sp. frustules for optical detection of lysozyme, Anal. Bioanal. Chem. 408 (2016) 2083-2093.

[30] X. Fu, L. Sheng, Y.S. Yu, M.H. Ma, Z.X. Cai, X. Huang, Rapid and universal detection of ovalbumin based on N,O,P-co-doped carbon dots-fluorescence resonance energy transfer technology, Sens. Actuators, B 269 (2018) 278-287.

[31] W.T. Hu, L.P. Ding, J.H. Cao, L.L. Liu, Y.T. Wei, Y. Fang, Protein binding-induced surfactant aggregation variation: a new strategy of developing fluorescent aqueous sensor for proteins, ACS Appl. Mater. Interfaces 7 (2015) 4728-4736.

[32] J.R. Wei, Y.L. Ni, W. Zhang, Z.Q. Zhang, J. Zhang, Detection of glycoprotein through fluorescent boronic acid-based molecularly imprinted polymer, Anal. Chim. Acta 960 (2017) 110-116.

[33] L. Tan, K.C. Chen, C. Huang, R.F. Peng, X.Y. Luo, R. Yang, Y.F. Cheng, Y.W. Tang, A fluorescent Turn-on detection scheme for $\alpha$-fetoprotein using quantum dots placed in a boronate-modified molecularly imprinted polymer with high affinity for glycoproteins, Microchim. Acta 182 (2015) 2615-2622.

[34] D.Y. Li, Y.P. Qin, H.Y. Li, X.W. He, W.Y. Li, Y.K. Zhang, A "Turn-on" fluorescent receptor for setecting tyrosine phosphopeptide using the surface imprinting procedure and the epitope approach, Biosens. Bioelectron. 66 (2015) 224-230. 